\title{
Improving hepatitis B vaccination rates for advanced chronic kidney disease patients: a quality improvement initiative
}

\author{
Priyanka Mysore ${ }^{1} \cdot$ Roaa M. Khinkar ${ }^{2,3}$ (1) Donna McLaughlin ${ }^{4} \cdot$ Sonali Desai ${ }^{3,5} \cdot$ Gearoid M. McMahon $^{4}$. \\ Catherine Ulbricht ${ }^{3} \cdot$ Mallika L. Mendu ${ }^{3,4}$
}

Received: 20 September 2020 / Accepted: 14 December 2020 / Published online: 7 January 2021

(C) Japanese Society of Nephrology 2021

\begin{abstract}
Introduction Chronic kidney disease (CKD) patients are vulnerable to hepatitis B, and immunization prior to end stage kidney disease is recommended to optimize seroconversion. Our institution undertook a process improvement approach to increase hepatitis B vaccination in stage 4 and 5 CKD patients.

Methods Four strategies were utilized such as: (1) Electronic health record (EHR)-based CKD registry to identify patients, (2) EHR-based physician/nurse reminders, (3) a co-located nurse appointment for vaccine administration, and (4) information sharing and provider awareness effort. The CKD registry was utilized to identify patients with stage 4 or 5 CKD, with at least two clinic visits in the prior 2 years, who had not received the hepatitis B vaccine or did not have serologic evidence of immunity. Target monthly vaccination rate was set at $75 \%$, based on clinic leadership, nephrologist, and nurse consensus. Results A total of 239 patients were included in the study period, from November 2018 to January 2019 (observation period) and from February 2019 to September 2019 (intervention period). Monthly vaccination rate improved from 48\% in November 2018 to the target rate of $75 \%$ by the end of the intervention (August and September 2019). There was a statistically significant increase from the rate of vaccination at a unique patient level in the first month of the baseline period, compared to the last month of the intervention period (51 vs. $75 \% p=0.03$ ).

Conclusions Utilizing a nurse-led approach to hepatitis B vaccination, coupled with EHR-based tools, along with continuous monitoring of performance, helped to improve hepatitis B vaccination among CKD stage 4 and 5 patients.
\end{abstract}

Keywords Hepatitis B vaccination · Chronic kidney disease · Nurse-driven initiative · Quality improvement

Priyanka Mysore and Roaa M. Khinkar are co-first authors.

Supplementary Information The online version contains supplementary material available at https://doi.org/10.1007/s1015 7-020-02013-4.

Roaa M. Khinkar

rkhinkar@bwh.harvard.edu; rkhinkar@kau.edu.sa

1 Department of Nephrology, Health Sciences Center, University of Manitoba, Winnipeg, MB, Canada

2 Department of Clinical Pharmacy, College of Pharmacy, King Abdulaziz University, Jeddah, Saudi Arabia

3 Department of Quality and Safety, Brigham and Women's Hospital, Harvard Medical School, One Brigham Circle, Second Floor, Office No. BC-2-WS 34, 1620 Tremont Street, Boston, MA 02120, USA

4 Renal Division, Brigham and Women's Hospital, Harvard Medical School, Boston, MA, USA

5 Rheumatology Division, Brigham and Women's Hospital, Harvard Medical School, Boston, MA, USA

\section{Introduction}

While cases of acute hepatitis B have declined since the peak in the early 1980s, preventing hepatitis B infection remains an important public health issue [1]. Since 1982, a significant effort has been made to reduce the risk of hepatitis B virus transmission and infection in the United States (US). The US Advisory Committee on Immunization Practices (ACIP) took further steps to ensure eradication of hepatitis $B$ virus transmission. In 1991, ACIP recommended universal hepatitis B vaccination in infants, focused on catch-up vaccination in highrisk children, as well as vaccination of high-risk adolescents and adults [2]. In 2017, the United States Centers for Disease Control (CDC) estimated that there were 20,900 acute cases of hepatitis B in the US [3]. Patients with CKD are particularly vulnerable to hepatitis $B$ due to impairment of cell-mediated and humoral immunity, impacting seroconversion [4]. Patients progressing to end stage kidney disease (ESKD) treated with 
hemodialysis are prone to infections related to blood product exposure [4]. In addition, it is well established that late stage CKD and ESKD patients in particular, have an impaired ability to seroconvert in the setting of vaccination [5], and that patients with earlier stages of CKD have improved seroconversion; thus, heightening the need for immunization at earlier stages of CKD [6]. Despite this evidence, vaccination strategies for kidney disease patients have typically targeted in-center hemodialysis units.

The ACIP recommends hepatitis B vaccination for (1) pre-dialysis patients, (2) dialysis patients, (3) potential kidney transplant recipients, and (4) diabetics over the age of 60, encompassing a substantial portion of patients in most CKD clinics $[3,7]$. Although there is a clear benefit to hepatitis B vaccination in CKD patients, only a few studies have been published regarding vaccination implementation strategies that can guide CKD clinics. Studies have shown that higher doses of hepatitis B vaccine, specifically $40 \mathrm{ug}$, lead to higher conversion rates $[6,8]$. An influenza and pneumococcal vaccination intervention at our institution targeted elderly and high-risk (those with asthma, human immunodeficiency virus (HIV), lung disease, and rheumatologic conditions) and demonstrated a significant increase in vaccination rates across specialties [9]. Other studies have implemented vaccination improvement strategies for zoster, pneumococcal, and influenza vaccination in patients with rheumatologic conditions and have demonstrated success $[10,11]$.

In 2016, our institution, along with others in our health system, developed a conceptual framework for optimizing care across the CKD spectrum that identified hepatitis B vaccination rates as a metric of interest [12]. Our institution undertook a process improvement approach with the goal of increasing hepatitis B vaccination rates by creating a novel nurse-driven administration algorithm based on analysis of current clinic workflow deficiencies. Our improvement work utilized four fundamental strategies such as: (1) utilizing an EHR-based CKD registry to identify patients meeting vaccination criteria, (2) prompting interdisciplinary communication through parallel EHR-based reminders, (3) maximizing workflow efficiency through co-located scheduled nurse appointments, and (4) promoting awareness through posted clinic information sheets and regular sharing of data. We sought to determine if this comprehensive data-driven quality improvement initiative would be successful in improving hepatitis B vaccination rates in our CKD clinic, specifically in patients with stage 4 and 5 CKD (estimated glomerular filtration rate (eGFR) $<30 \mathrm{ml} / \mathrm{min} / \mathrm{m}^{2}$ ).

\section{Materials and methods}

\section{Study setting}

Brigham and Women's Medical Specialities Renal Clinic provides care to approximately 2000 general nephrology patients and kidney transplant recipients. There are 32 nephrologists, 8 nephrology fellows, 2 nephrology nurses, 4 nephrology/pancreas transplant nurses, and administrative staff. All patient data and medication orders are completed through the EHR, EPIC Partners version. Prior to the intervention period, there was no standardized process to provide hepatitis B virus vaccination for CKD patients. An observation period was from November 1, 2018 to January 31, 2019; the intervention period was from February 1, 2019 to September 30, 2019. Nephrologists were expected to identify patients during their routine follow-up visits and order hepatitis B vaccinations as appropriate.

\section{Quality metric development}

The clinic utilizes a CKD registry to facilitate comprehensive CKD care improvement [13]. Utilizing this registry, we were able to identify CKD patients who had not received the hepatitis $B$ vaccine or did not have serologic evidence of immunity to hepatitis B (hepatitis B surface antibody (HBsAb) greater than $>12 \mathrm{mIU}$ ). Several quality control checks, including manual verification of patient lists, were conducted by our team to ensure data integrity. The metric denominator was defined as patients with CKD stage 4 or 5 (eGFR $<30 \mathrm{ml} / \mathrm{min} / \mathrm{m}^{2}$, not on dialysis), with at least two visits in the nephrology clinic in the last 24 months. The metric numerator was defined as patients who did have a documented hepatitis $B$ vaccine or a seroprotective HBsAb titre $>12 \mathrm{mIU}$. Performance on the hepatitis B vaccination quality metric was monitored on a monthly basis. Overall clinic rates, both cumulative and monthly rates of eligible patients receiving vaccination, and provider-specific rates were shared with clinic leadership. A target monthly vaccination rate was set at $75 \%$ based on clinic leadership, nephrologist, and nurse consensus; this target was chosen as it was thought to be feasible, and reflective of patients who may refuse vaccination or have other contraindications. Hepatitis B vaccination rates were assessed both at a visit level and a unique patient level. Data at a visit level account for the fact that some patients had more than one visit during the study period, and monthly and cumulative data are presented at the visit level (the percentage of visits meeting vaccination criteria). Unique patient level data were also assessed to examine rates of vaccination among unique patients. 


\section{Pre-intervention Workflow}

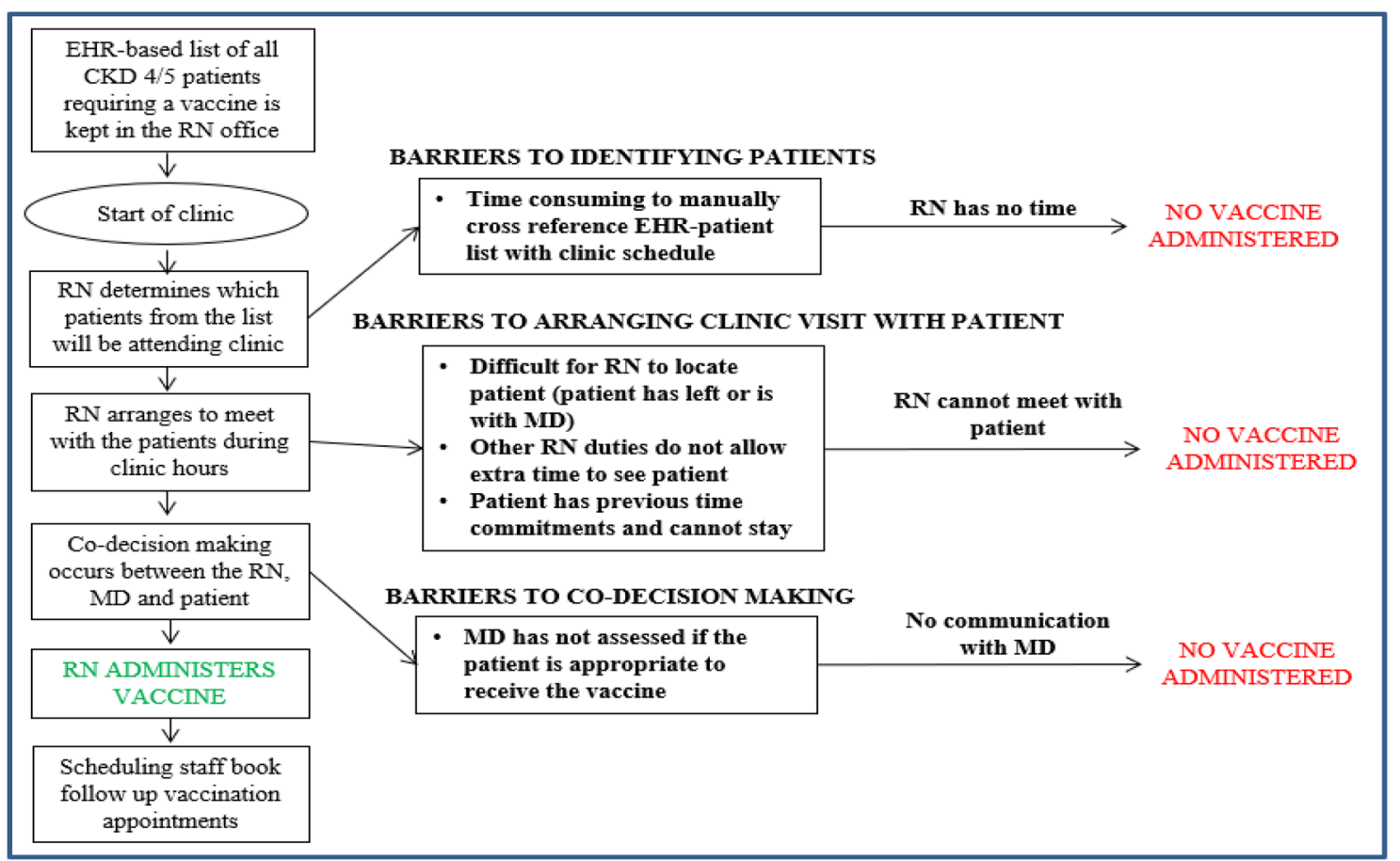

\section{Post-intervention Workflow}

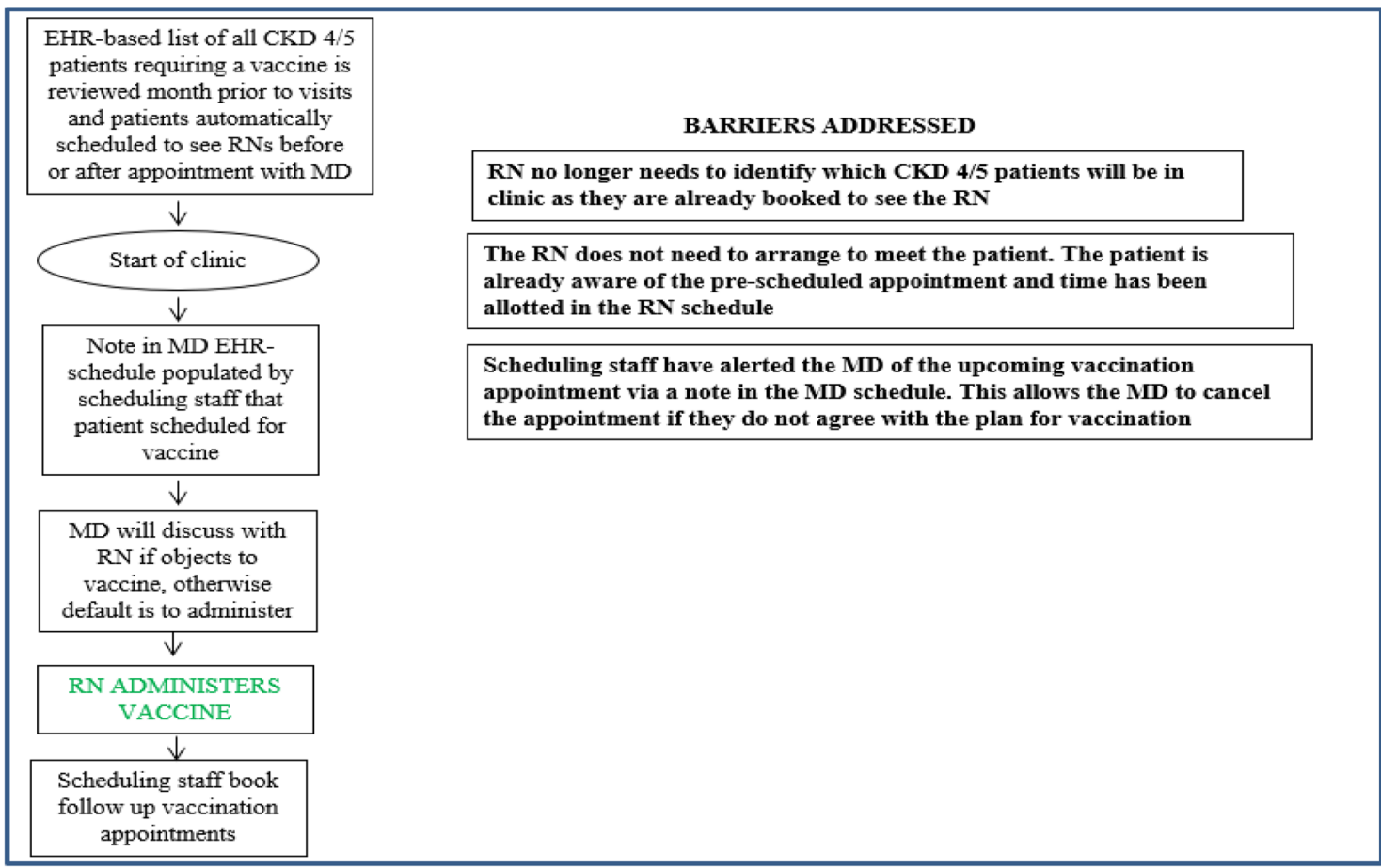

Abbreviations: $E H R$, electronic health record; $R N$, registered nurse; $M D$, medical doctor

Fig. 1 Pre- and post-intervention workflow and barriers addressed 


\section{Assessment of barriers}

We utilized several quality-improvement methods to understand causes of suboptimal vaccination rates, including focus groups, nurse interviews, and process mapping as shown in Fig. 1. Three key barriers to vaccination were identified such as: (1) nurses identifying patients who required a vaccine by cross referencing the CKD registry with clinic schedules, (2) nurses needing to arrange a scheduled clinic visit to discuss vaccination, and 3 ) nurses ensuring co-decision-making between the physician, patient, and administering nurse. Our intervention was developed to strategically address these barriers. There were no challenges with implementation due to insurance coverage or cost of the vaccine.

\section{Interventions}

\section{Promoting awareness}

For the overall intervention, a logo and short title (The 'B prepared' Initiative) (as shown in Fig. 2) were created to facilitate discussion and establish expectations. Information about the study was circulated through several venues such as: a presentation at multiple divisional rounds, clinic-wide memos, individual physician emails, and workroom posters.

\section{Algorithm implementation, co-located nursing appointments, EHR-visit notes}

A list of patients meeting the numerator and denominator criteria was generated using the CKD data registry; patients were categorized based on provider and next visit date for ease of use. The clinic scheduling staff utilized the list to book

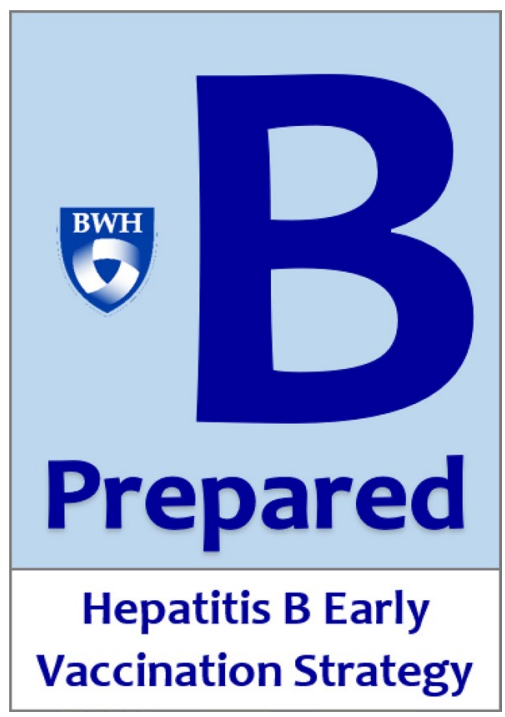

Fig. 2 The B prepared Initiative Logo patients for a nurse clinic visit on the day of their next physician visit. The scheduling staff also entered a note in the physician schedule that informed the physician of the patient's upcoming nurse clinic visit. On the day of the patient clinic visit, notes appeared in both the physician and nurse schedules informing them that the patient was scheduled for a nurse visit to discuss hepatitis B vaccination. The prompt allowed discussion between the physician and patient regarding vaccine appropriateness. The patient then completed the nurse visit for the vaccination. Subsequent follow-up for completion of the hepatitis B vaccination series was arranged by the nurse in conjunction with the scheduling staff. All but one of the nephrologists allowed their patients to participate in the ordering algorithm.

\section{Iterative changes to process}

Utilizing a Plan-Do-Study-Act (PDSA) cycle, we implemented a number of iterative changes to the original process. First, there were a number of patients who were booked for their nurse visit without a HBsAb titre. Subsequently, after physician input, all patients required a baseline titre prior to vaccination. Second, based on nurse, patient, and physician preference, some patients were scheduled for the nurse visit $30 \mathrm{~min}$ ahead of the physician visit, when there was an identified need for a 30-min discussion about the importance of hepatitis B vaccination. Finally, there were periods of administrative scheduling staff turnover that required co-author RK to assist with scheduling.

\section{Study design and analysis}

We conducted a quasi-experimental study design, implementing a quality improvement initiative and capturing outcome measures pre-and post-intervention. The primary outcome measure was the percentage of total clinic patients (at a visit level) meeting the hepatitis B vaccination metric as defined by the numerator and denominator above; both cumulative and monthly rates were tracked. Calculation of proportions was conducted using Microsoft Excel (version 2016), and chi-squared test for comparison of proportions was calculated using Statistical Analysis System (SAS), (version 9.4).

Baseline data were included from November 1, 2018 to January 31, 2019. Institutional review board approval was not required as this study met the requirements of a qualityimprovement initiative.

\section{Results}

During the baseline observation and intervention period, 239 stage 4 and 5 CKD patients were included (Table 1), accounting for 558 overall visits. Of 239, 29 patients had 
Table 1 Patient demographics and clinical characteristics of stage 4 and 5 CKD patients included in the hepatitis $\mathrm{B}$ vaccination process improvement study

\begin{tabular}{|c|c|}
\hline Patient characteristics & $N, \%(N=239)$ \\
\hline Median age [IQR] & $71[62,77]$ \\
\hline \multicolumn{2}{|l|}{ CKD Stages/eGFR } \\
\hline CKD Stage 4 & $193(80.8)$ \\
\hline CKD Stage 5 & $46(19.2)$ \\
\hline Median eGFR $\mathrm{mL} / \mathrm{min} / \mathrm{m}^{2}[\mathrm{IQR}]$ & $10[16,26]$ \\
\hline \multicolumn{2}{|l|}{ Gender } \\
\hline Female & $124(51.9)$ \\
\hline Male & $115(48.1)$ \\
\hline \multicolumn{2}{|l|}{ Language } \\
\hline English & $210(87.9)$ \\
\hline Spanish & 19 (7.9) \\
\hline Other & $10(4.2)$ \\
\hline \multicolumn{2}{|l|}{ Race } \\
\hline White & $155(64.9)$ \\
\hline Black & $51(21.3)$ \\
\hline Hispanic & $9(3.8)$ \\
\hline Asian & $5(2.1)$ \\
\hline Other & $19(7.9)$ \\
\hline \multicolumn{2}{|l|}{ Marital status } \\
\hline Married/civil union & $136(56.9)$ \\
\hline Single & $61(25.5)$ \\
\hline Divorced & $15(6.3)$ \\
\hline Other & $27(11.3)$ \\
\hline \multicolumn{2}{|l|}{ Educational level } \\
\hline Graduated-College & $76(31.8)$ \\
\hline Graduated—High school & $51(21.3)$ \\
\hline Graduated-Post graduate & $25(10.4)$ \\
\hline Other & $87(36.4)$ \\
\hline \multicolumn{2}{|l|}{ Insurance type } \\
\hline Medicare & $136(56.9)$ \\
\hline Other & $103(43.1)$ \\
\hline \multicolumn{2}{|l|}{ Comorbidities } \\
\hline Hypertension & $204(85.4)$ \\
\hline Diabetes & $109(45.6)$ \\
\hline Coronary artery disease & $67(28.0)$ \\
\hline Cancer & $50(20.9)$ \\
\hline Peripheral vascular disease & $31(13.0)$ \\
\hline Chronic obstructive pulmonary disease & $23(9.6)$ \\
\hline Cerebrovascular accident & $21(8.8)$ \\
\hline Congestive heart failure & $21(8.8)$ \\
\hline Lupus & $8(3.3)$ \\
\hline
\end{tabular}

$I Q R$ interquartile range

visits in the pre-intervention period only, 111 patients had their visits in the post-intervention period only, and 99 patients had the visits in the pre-and-post-intervention periods. The median age was 71 years, and $52 \%$ were female.
Among the cohort, 52\% were White, 21\% Black, and 9\% Hispanic; $57 \%$ were married and $32 \%$ graduated college.

Of the 239 patients included during the study period, 135 patients (56\%) met metric criteria for vaccination, of which 84 completed 3 vaccinations and 33 demonstrated seroconversion on initial testing. Among the 84 patients who completed 3 vaccinations, 33 demonstrated seroconversion on follow-up testing. Approximately $13 \%$ of patients did not complete the hepatitis B vaccination protocol due to scheduling issues.

Both monthly and cumulative data reflected on Figs. 3 and 4 were at a visit level. During the three-month baseline period from November 2018 to January 2019, the monthly vaccination rate was between 48 and 54\% (see Fig. 3). An initial lag was observed in the first few months of the intervention roll out; the monthly vaccination rate was between 52\% in February 2019 and 46\% in April 2019. Improvement was seen starting in May 2019 with a monthly vaccination rate of $63 \%$, and the target vaccination rate of $75 \%$ was achieved during the last two months of the intervention (August 2019, 80\% and September 2019, 75\%).

There was improvement in the cumulative vaccination rate during the intervention period; the vaccination rate increased from 18\% in February 2019 to 58\% in September 2019 (see Fig. 4). At a unique patient level, there was a statistically significant increase from the rate of vaccination in November 2018 (51\% of unique patients), the first month of the baseline period, to September 2019, the last month of the intervention period ( $75 \%$ of unique patients), $p=0.03$.

\section{Discussion}

Our study demonstrates that after the implementation of a practical hepatitis B vaccination strategy in the ambulatory setting, there was an increase in hepatitis B vaccination rates in stage 4 and 5 CKD patients. We found that the algorithmbased approach reduced the burden of work for both nephrologists and nurses, with minimal change in work burden for the scheduling staff. This automated approach was feasible to implement and led to sustainable improved monthly rates of vaccination.

This study is unique given it is focused on improving hepatitis B vaccination in CKD patients. Other studies have illustrated that earlier hepatitis B vaccination for CKD patients leads to improved seroconversion [14], or demonstrated improved rates of heart failure-related hospitalization in patients receiving flu vaccination [15]. Ishigami et al. demonstrated the cost effectiveness of pneumococcal vaccine in CKD patients, and found that vaccination was cost effective for patients aged 50-64 and 65-79 years, (US \$38,000/quality-adjusted life-year (QALY)) and (US $\$ 15,000 /$ QALY), respectively [16]. A number of studies 
Fig. 3 Monthly rates of stage 4 and 5 CKD patients who have documented hepatitis B immunity or completed hepatitis B vaccination during observation and intervention periods
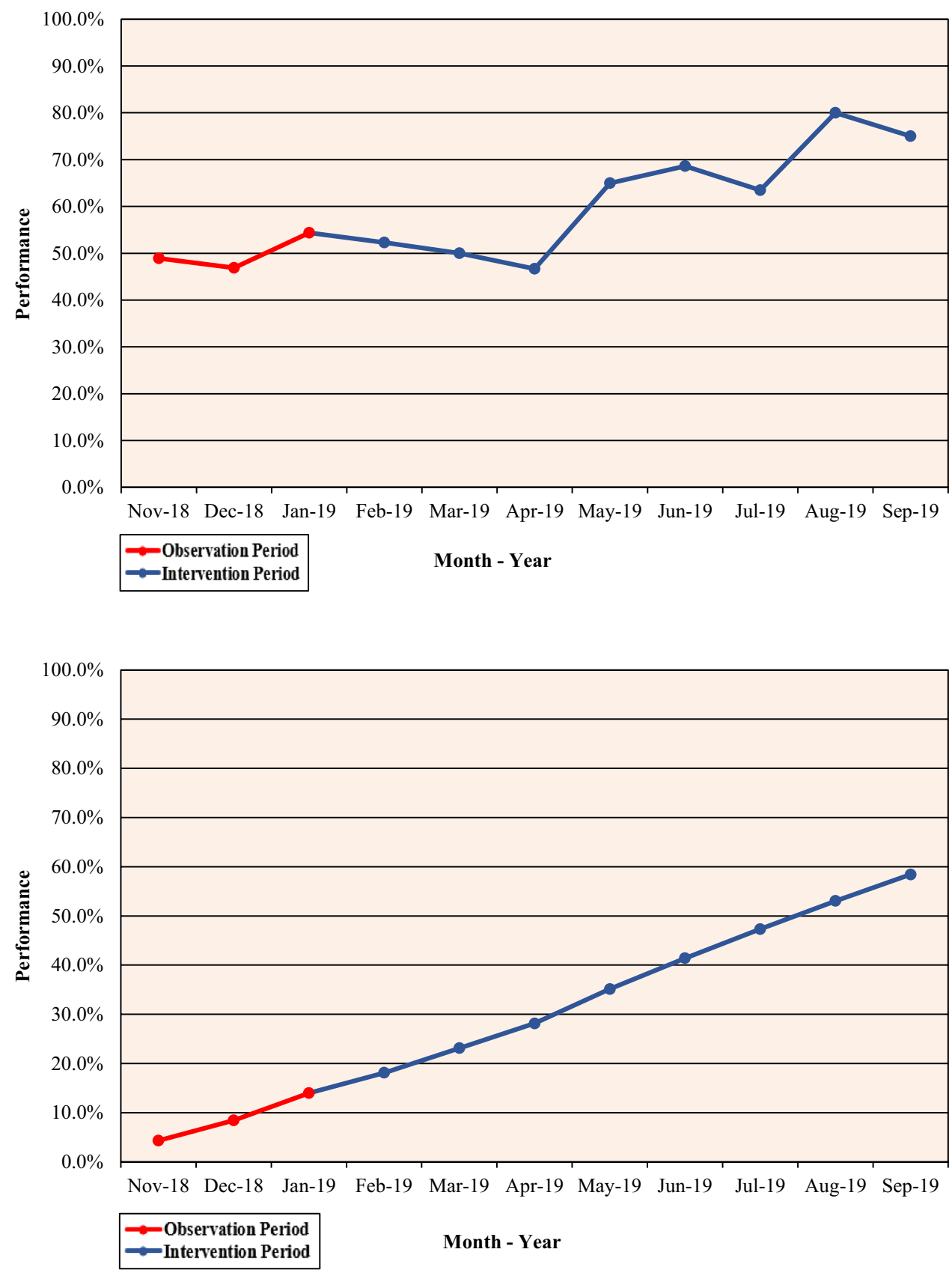

Fig. 4 Cumulative clinic performance rate of stage 4 and 5 CKD patients who have documented hepatitis B immunity or completed hepatitis B vaccination during observation and intervention periods have explored vaccination strategies in high-risk patients and incorporated ordering algorithms, electronic alerts, physician reminders, patient letters, and nurse-led efforts [9, 10]. Baker et al. focused on improving influenza, pneumococcal, and herpes zoster vaccination rates in rheumatoid arthritis patients by implementing electronic alerts along with linked order sets, patient outreach, frequent reports, and feedback to physicians; although the rate of influenza vaccination remained unchanged, pneumococcal and zoster vaccination rates increased from 28.7 to 45.8 and from 2.5 to $4.5 \%$, respectively [10]. Pennant et al. found that physician reminders and patient letters, coupled with a nurse-driven model, significantly improved rates of pneumococcal and influenza vaccination in patients with asthma, HIV, chronic lung disease, or on immunosuppressive medications for rheumatoid arthritis [9].

The dearth of evidence regarding hepatitis $\mathrm{B}$ CKD vaccination initiatives may reflect the unique challenge in the administration of the hepatitis B vaccine itself; in particular, the multitude of steps are needed to complete vaccination. Patients are often unsure of whether they received the vaccine or not. Thus, HBsAb titers are often needed to confirm vaccination. A series of three vaccines are needed, with the second and third vaccines typically administered 1 and 
5 months after the initial vaccination. Therefore, patients need multiple visits to complete the second and third dose series of the vaccine.

Our study was sustained over an 8-month period but was paused due to an unexpected reduction in clinic nursing coverage. Ideally, the intervention would have continued as a long-term intervention. We were in the process of relaunching the intervention when the coronavirus 2019 (COVID19) pandemic began in February 2020. The pandemic has prompted a rapid shift toward virtual outpatient care to minimize patient travel and promote physical distancing. This introduces further challenges to the administration of vaccinations. Considering this new virtual model of care, an algorithm-based approach to vaccination is particularly important in structuring and coordinating patient visits. We anticipate adapting our model to focus on synchronizing hepatitis vaccinations with necessary in-person clinic visits and other vaccinations (i.e., influenza vaccination).

There are several limitations to our study. First, we did not mandate repeating titers after vaccination, nor did we standardize how to assess non-responders. We see this as an opportunity for another iteration of this initiative, now that we have established efficacy with this approach. Second, we had important resources, specifically an EHR-based CKD registry and motivated nurses. The approach is less generalizable in more resource -constrained settings, though some of the general principles are likely applicable. Third, our study did not have rigorous qualitative data, for instance, patient experience survey data. Fourth, we had a limited period of observation and intervention and a limited sample size, which restricted our ability to perform more complex statistical analyses such as an interrupted time-series regression. Finally, our study was focused on hepatitis B vaccine, and in a future iteration, we recognize the opportunity to incorporate influenza, pneumococcal, and zoster vaccines.

There are a number of strengths to highlight. The study involved a large general CKD clinic with a fairly large number of nephrologists with varied practices. The data were rigorously tracked using a validated EHR-based CKD registry [13]. We performed a methodical assessment of workflow and barriers to implementation, and iteratively refined our quality improvement approach. We ensured that there was minimal additional workflow for staff at all levels and emphasized convenience for patients.

Our intervention capitalized on the existence of a patient data registry. The use of electronic data registries is becoming more common in the ambulatory setting and has been shown to improve the compliance with quality metrics [17, 18]. Utilizing population-based interventions such as this one creates structured approaches to quality-improvement initiatives that can be expanded and generalized to other settings. The utilization of data registries to measure adherence to quality metrics at an individual patient level presents a huge opportunity to advance and customize quality of care delivered; particularly, in the ambulatory setting that has been traditionally understudied.

\section{Conclusions}

In summary, the findings of our study demonstrate a practical, algorithm-based, nurse-driven approach to improve the rates of hepatitis $B$ vaccination among stage 4 and 5 CKD patients. Yet, the current COVID-19 situation necessitates further modification of the implemented strategy to ensure a practical and sustainable strategic approach.

Acknowledgements We acknowledge the immense contributions of Laura Judge NP, in the design and implementation of the study.

Author contribution Drs. Mysore, Desai, McMahon, Mendu and Ms. Khinkar and McLaughlin contributed to the study concept and design. Dr. Mysore and Ms. Khinkar contributed to acquisition of data. All authors contributed to analysis and interpretation of data, drafting of the manuscript and have approved the final version.

Availability of data and material Drs. Mysore and Mendu, and Ms. Khinkar had full access to all the data in the study and take responsibility for the integrity of the data and the accuracy of the data analysis.

\section{Compliance with ethical standards}

Conflict of interest Dr. Mendu provides consulting service to Bayer $\mathrm{AG}$, unrelated to this manuscript.

Ethical approval Institutional review board (IRB) approval was not required as this study met the requirements of a quality-improvement initiative.

Consent for publication The results presented in this paper have not been published previously in whole or part.

\section{References}

1. Harris AM, Iqbal K, Schillie S, Britton J, Kainer MA, Tressler S, Vellozzi C. Increases in acute hepatitis B virus infections-kentucky, tennessee, and west virginia, 2006-2013. MMWR Morb Mortal Wkly Rep. 2016;65:47-50.

2. Centers for disease control and prevention. Achievements in public health: hepatitis B vaccination --- United States, 1982--2002. 2002. https://www.cdc.gov/mmwr/preview/mmwrhtml/mm512 5a3.htm. Accessed 27 Oct 2020.

3. Centers for disease control and prevention. Hepatitis B questions and answers for health professionals. 2020. https://www.cdc.gov/ hepatitis/hbv/hbvfaq.htm. Accessed 11 May 2020

4. Janus N, Vacher L-V, Karie S, Ledneva E, Deray G. Vaccination and chronic kidney disease. Nephrol Dial Transplant. 2008;23:800-7.

5. DaRoza G, Loewen A, Djurdjev O, Kiaii M, Taylor P, Levin A. Stage of chronic kidney disease predicts seroconversion after hepatitis B immunization: earlier is better. Am J Kidney Dis. 2003;42:1184-92. 
6. Ghadiani MH, Besharati S, Mousavinasab N, Jalalzadeh M. Response rates to HB vaccine in CKD stages 3-4 and hemodialysis patients. J Res Med Sci. 2012;17:527-33.

7. Danziger-Isakov L, Kumar D. Vaccination in solid organ transplantation. Am J Transplant. 2013;13:311-7.

8. Agarwal SK, Irshad M, Dash SC. Comparison of two schedules of hepatitis B vaccination in patients with mild, moderate and severe renal failure. J Assoc Physicians India. 1999;47:183-5.

9. Pennant KN, Costa JJ, Fuhlbrigge AL, Sax PE, Szent-Gyorgyi LE, Coblyn J, Desai SP. Improving influenza and pneumococcal vaccination rates in ambulatory specialty practices. Open Forum Infect Dis. 2015;2:ofv19.

10. Baker DW, Brown T, Lee JY, Ozanich A, Liss DT, Sandler DS, Ruderman EM. A multifaceted intervention to improve influenza, pneumococcal, and herpes zoster vaccination among patients with rheumatoid arthritis. J Rheumatol. 2016;43:1030-7.

11. Sheth H, Moreland L, Peterson H, Aggarwal R. Improvement in herpes zoster vaccination in patients with rheumatoid arthritis: a quality improvement project. J Rheumatol. 2017;44:11-7.

12. Mendu ML, Waikar SS, Rao SK. Kidney disease population health management in the era of accountable care: a conceptual framework for optimizing care across the CKD spectrum. Am J Kidney Dis. 2017;70:122-31.

13. Mendu ML, Ahmed S, Maron JK, Rao SK, Chaguturu SK, May MF, Mutter WP, et al. Development of an electronic health recordbased chronic kidney disease registry to promote population health management. BMC Nephrol. 2019;20:72.
14. Grzegorzewska AE. Hepatitis B vaccination in chronic kidney disease: review of evidence in non-dialyzed patients. Hepat Mon. 2012;12:e7359.

15. Fang Y-A, Chen C-I, Liu J-C, Sung L-C. Influenza vaccination reduces hospitalization for heart failure in elderly patients with chronic kidney disease: a population-based cohort study. Acta Cardiol Sin. 2016;32:290-8.

16. Ishigami J, Padula W, Grams ME, Chang AR, Jaar B, Gansevoort RT, Bridges JFP, et al. Cost-effectiveness of pneumococcal vaccination among patients with CKD in the United States. Am J Kidney Dis. 2019;74:23-35.

17. Navaneethan SD, Jolly SE, Schold JD, Arrigain S, Saupe W, Sharp J, Lyons J, et al. Development and validation of an electronic health record-based chronic kidney disease registry. Clin J Am Soc Nephrol. 2011;6:40-9.

18. Jolly SE, Navaneethan SD, Schold JD, Arrigain S, Konig V, Burrucker YK, Hyland J, et al. Development of a chronic kidney disease patient navigator program. BMC Nephrol. 2015;16:69.

Publisher's Note Springer Nature remains neutral with regard to jurisdictional claims in published maps and institutional affiliations. 